\title{
Discussion on Reciprocity, Unitary Matrix, and Lossless Multiple Beam Forming Networks
}

\author{
Nelson Jorge G. Fonseca \\ Moltek Consultants Ltd. for the European Space Agency (ESA), Antenna and Sub-Millimetre Wave Section, Keplerlaan 1, P.O. Box 299, \\ $2200 A G$ Noordwijk, Netherlands
}

Correspondence should be addressed to Nelson Jorge G. Fonseca; nelson.fonseca@esa.int

Received 15 December 2014; Revised 19 March 2015; Accepted 20 March 2015

Academic Editor: Yuan Yao

Copyright (C) 2015 Nelson Jorge G. Fonseca. This is an open access article distributed under the Creative Commons Attribution License, which permits unrestricted use, distribution, and reproduction in any medium, provided the original work is properly cited.

\begin{abstract}
The Lorentz reciprocity theorem enables us to establish that the transmitting and receiving patterns of any antenna are identical, provided some hypotheses on this antenna and the surrounding medium are satisfied. But reciprocity does not mean that the antenna behaves the same in the transmitting and the receiving modes. In this paper, array antennas fed by multiple beam forming networks are discussed, highlighting the possibility to have different values of internal losses in the beam forming network depending on the operation mode. In particular, a mathematical condition is derived for the specific case of a multiple beam forming network with lossless transmitting mode and lossy receiving mode, such a behavior being fully consistent with the reciprocity theorem. A theoretical discussion is provided, starting from a simple 2-element array to a general $M \times N$ multiple beam forming network. A more practical example is then given, discussing a specific $4 \times 8$ Nolen matrix design and comparing theoretical aspects with simulation results.
\end{abstract}

\section{Introduction}

The Lorentz reciprocity theorem enables us to establish that the transmitting and receiving patterns of any antenna are identical, provided some hypotheses on this antenna, the probe antenna used to evaluate the patterns and the medium in between them are satisfied. This is quite a simple and natural property. Still, its interpretation is not always straightforward. This difficulty is well illustrated by recent discussions on the modeling of receiving antennas and the distinction between its absorbed and scattered powers [15]. Recent work presented by the author has also raised discussions on this matter in connection with multiple beam forming network properties [6, 7]. Indeed, it was found that some beam forming networks exhibit an interesting property: they are theoretically lossless in the transmitting mode but have losses in the receiving mode which depend on the incidence angle of the incoming plane wave. Without any additional explanation, one might think that this property violates the reciprocity theorem. Interestingly enough, this property can be related to the one described in [8] in the case of nonsymmetric propagation scenarios: reciprocity holds between two antennas separated by a nonsymmetric propagation medium even if the path loss variations as a function of range along a given propagation path are totally different for the forward and backward scenarios. Clearly, reciprocity does not mean that the field distribution has to be the same in the transmitting and the receiving modes but only mean that the ratio of transmitted to received field strengths between the two considered antennas, when treated as a twoport network, is the same when the roles of transmitter and receiver are interchanged, provided the considered antennas and medium in which they are placed are linear. Having this in mind, a beam forming network with insertion loss values different in the transmitting and receiving modes is possible as long as this loss difference is compensated for somewhere else in the link budget between the two considered antenna ports. In this paper, we discuss the theoretical bases required to understand this peculiar property. We introduce the problem with a simple example: a two-element linear array fed by two types of power dividers. Then, we address the general case of $M \times N$ multiple beam forming networks, setting the link between the scattering matrix properties and a theoretically lossless operation mode. We conclude this paper 
discussing a practical example previously introduced in the literature and related to this theoretical aspect.

\section{Hypotheses and Problem Description}

2.1. Calculation Hypotheses. Before starting with a specific simple case to illustrate the difference in behavior between transmitting and receiving array antennas, let us first introduce the hypotheses. An important application of the Lorentz reciprocity theorem is the comparison between the receiving and transmitting patterns of an antenna. Let us consider two distinct antennas, labeled 1 and 2 . First, antenna 1 transmits while antenna 2 receives, and second, antenna 2 transmits while antenna 1 receives [9]. Treating these two-antenna system as a two-port network, each port representing one of the two antennas, it can be shown that the mutual impedances between these two ports are identical $\left(Z_{12}=Z_{21}\right)$. As we focus in this paper on beam forming network properties, we prefer to use this result translated into scattering parameters; that is, $S_{12}=S_{21}$ [10]. The main consequence of this relation is that the transmitting and receiving patterns of an antenna are identical, provided the two considered antennas are in a linear and isotropic medium [9]. To simplify the calculations, we assume that the distance between the two antennas is much longer than the wavelength. Consequently, the behavior of the antenna investigated in the transmitting mode can be derived from its far-field radiation pattern, while in the receiving mode an incident plane wave is assumed [11, 12]. Furthermore, we assume all the elementary antennas to be isotropic. Consequently, the effective aperture in receive is assumed to be the same for all the elementary antennas and is independent on the incident angle, resulting in a common multiplicative coefficient neglected in the following results. Similarly, the elementary antenna gain is assumed to be equal to one in any angular direction in the transmitting mode. This is consistent with the fact that reciprocity lies on relative values in linear medium, and thus directivity normalization at array element level does not affect our conclusions. The elementary antennas are considered perfectly matched and lossless: in the transmitting mode, all the power provided in guided-wave at the antenna port is radiated as a free-space wave and reciprocally, all the incident power captured by the antenna as a free-space wave is converted into a guided-wave delivered to the antenna port in the receiving mode. Additionally, the beam forming networks considered are all based on passive components, contain no anisotropic materials, and are fully characterized using only the fundamental mode at each port. In these conditions, reciprocity applies at beam forming network level; that is, $S_{i j}=S_{j i}$, with $i$ and $j$ being two integers comprised between 1 and $P$ in the case of a $P$-port device. With these hypotheses, the scattering matrix $[S]$ of the beam forming network is necessarily symmetric [10]. In this paper, the notation $V_{p}^{+}$is used for the amplitude of the voltage wave incident on port $p$ while $V_{p}^{-}$is the amplitude of the voltage wave reflected from port $p$, related by the following equation [10]:

$$
\left[V^{-}\right]=[S]\left[V^{+}\right]
$$

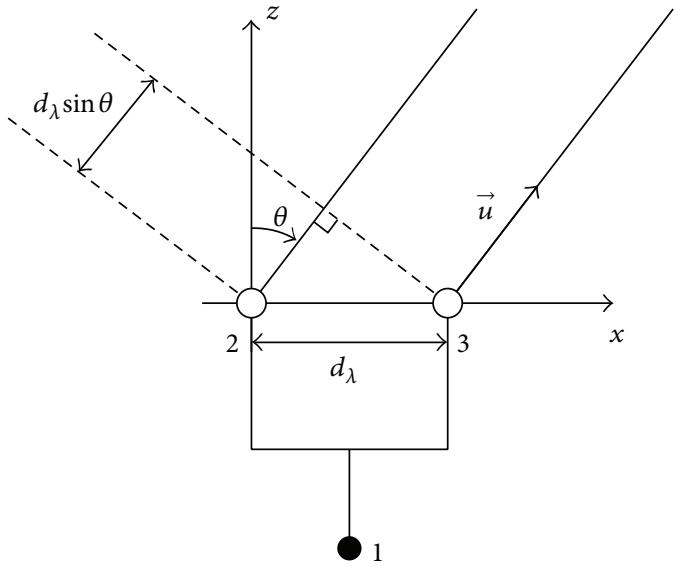

FIGURE 1: 2-element linear array parameters definition.

where $\left[V^{+}\right]$and $\left[V^{-}\right]$are the two vectors containing the values of $V_{p}^{+}$and $V_{p}^{-}$for $p$ varying from 1 to $P$.

2.2. Problem Description through a Simple Case. To introduce the property discussed in the introduction, we consider a 2 element linear array with the notations defined in Figure 1. The array antenna has one beam port, referred to as port 1 , and two array element ports, referred to as ports 2 and 3 . The beam forming network is a 3-port junction. It can be demonstrated that such a device cannot be simultaneously lossless and matched at all ports [13]. But what is meant by lossless? And how this property may depend on the operation mode of the antenna, either transmitting or receiving? Actually, do we really need all ports to be matched when such a device is used as a beam forming network? To answer these questions, let us consider two technical solutions for the proposed 3-port junction: a tee junction and a Wilkinson power divider [13]. The scattering matrix of a tee junction is the following:

$$
[S]=\frac{-1}{2 \sqrt{2}}\left(\begin{array}{ccc}
0 & i 2 & i 2 \\
i 2 & -\sqrt{2} & \sqrt{2} \\
i 2 & \sqrt{2} & -\sqrt{2}
\end{array}\right) .
$$

This matrix is unitary because the following relation is verified:

$$
[S]^{t}[S]^{*}=[I]
$$

where $[S]^{t}$ and $[S]^{*}$ are, respectively, the transpose and conjugate of $[S]$ and $[I]$ is a unit (or identity) matrix.

This property is a necessary and sufficient condition to have a so-called lossless network, that is, a network that has no internal power dissipation whatever the input power distribution applied to any combination of its ports [10] (typical line losses related to a practical implementation of such components are neglected in this theoretical description). But what a lossless network means from an antenna point of view? Is all the power delivered at the antenna port radiated? And reciprocally, is all the power captured by the antenna transmitted to the antenna port? Let us see what happens with the tee junction. We consider a unitary incident voltage wave 


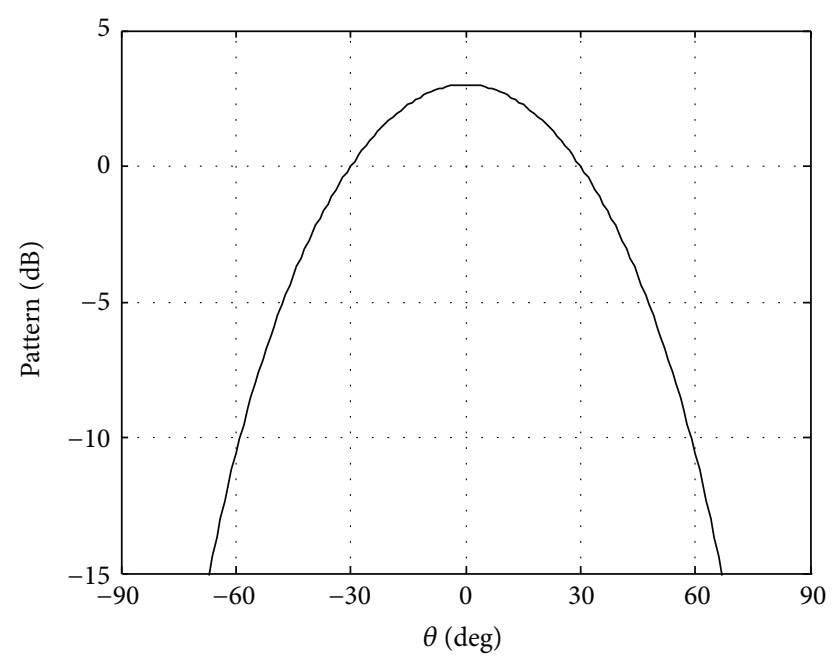

FIgURE 2: Radiation pattern of a 2-element linear array.

at the antenna port $\left(V_{1}^{+2}=1\right)$. Using $(2)$, the voltage wave vector reflected is

$$
[S]\left[\begin{array}{l}
1 \\
0 \\
0
\end{array}\right]=\frac{-i}{\sqrt{2}}\left[\begin{array}{l}
0 \\
1 \\
1
\end{array}\right] .
$$

All the power supplied at the antenna port is delivered to the array element ports because $\left|V_{2}^{-}\right|^{2}+\left|V_{3}^{-}\right|^{2}=1$. The radiation pattern in a plane containing the linear array can be obtained using the following array factor formula [14]:

$$
E(\theta)=V_{2}^{-}+V_{3}^{-} e^{j 2 \pi d_{\lambda} \sin \theta},
$$

where $d_{\lambda}$ is the distance normalized to the wavelength between the two array elements.

In Figure 2, this radiation pattern is illustrated for the specific case $d_{\lambda}=0.5$, which simplifies the mathematical evaluation of the array directivity [14]. A more rigorous evaluation of the array directivity as a function of $d_{\lambda}$ is possible, but the specific case considered here is sufficient to demonstrate the peculiar property this paper purposes to highlight. Figure 2 indicates a gain of $3 \mathrm{~dB}$ in the normal direction over the array element pattern. The radiation in the angular direction $\theta=30^{\circ}$ is reduced to $0 \mathrm{~dB}$. As all the power delivered at the antenna port is available at the array element ports, the transfer scattering parameter between the two antenna ports of the two-antenna system considered is directly given by the array gain and is then depending on the observation angle (which can be considered as the angular position of antenna 2). The values achieved, higher than $0 \mathrm{~dB}$ for some angular directions, are due to the simplifying hypotheses and corresponding normalization and should not be regarded as effective transfer coefficient values.

Now, we consider the receiving mode assuming an incident plane wave. The signals captured by the array elements depending on the incidence angle $\theta$ are

$$
\begin{aligned}
V_{2}^{+} & =1, \\
V_{3}^{+} & =e^{j 2 \pi d_{\lambda} \sin \theta} .
\end{aligned}
$$

Interestingly, the power received at each array element does not depend on the incidence angle (only the relative phase between the two received voltages varies with the incidence angle) and is equivalent to a gain of $3 \mathrm{~dB}$, which is the maximum gain observed in the transmitting mode, reached in the broadside direction. Using (2) and (6), the voltage wave vector reflected in the receiving mode is

$$
[S]\left[\begin{array}{c}
0 \\
1 \\
e^{j 2 \pi d_{\lambda} \sin \theta}
\end{array}\right]=\left[\begin{array}{c}
\frac{-i}{\sqrt{2}}\left(1+e^{j 2 \pi d_{\lambda} \sin \theta}\right) \\
\frac{1}{2}\left(1-e^{j 2 \pi d_{\lambda} \sin \theta}\right) \\
-\frac{1}{2}\left(1-e^{j 2 \pi d_{\lambda} \sin \theta}\right)
\end{array}\right] .
$$

One can note that $V_{1}^{-}$is equal to the array factor derived in the transmitting mode, as defined by (5). This is consistent with the reciprocity theorem as it brings the equality $S_{12}=$ $S_{21}$ for the two-antenna system. But this also means that the signal captured by the array elements is not always fully transmitted to the antenna port, as some may think from the "lossless beam forming network" appellation. In fact, part of the signal is directly reflected due to the mismatch at ports 2 and 3 and another part of the signal entering one of these two ports is reradiated by the other one due to the poor isolation between these two ports $\left(S_{23}\right)$. The phase difference between these two contributions varies with the incidence angle. For a normal incidence, no power is reradiated by ports 2 and 3 due to a destructive interference between these two contributions.

Let us consider now a Wilkinson power divider. Its scattering matrix is defined as follows:

$$
[S]=\frac{-i}{\sqrt{2}}\left(\begin{array}{lll}
0 & 1 & 1 \\
1 & 0 & 0 \\
1 & 0 & 0
\end{array}\right) .
$$

This component requires internal loads to enable matching of all ports $\left(S_{i i}=0\right)$. This matrix is not unitary. Interestingly, this component behaves exactly as the tee junction in the transmitting mode. This means that although the component matrix is not unitary, it is lossless in the transmitting mode. As the array geometry is unchanged, the incoming signals in the receiving mode are also defined by (6). Using (6) and (8), the voltage wave vector reflected in the receiving mode is

$$
[S]\left[\begin{array}{c}
0 \\
1 \\
e^{j 2 \pi d_{\lambda} \sin \theta}
\end{array}\right]=\frac{-i}{\sqrt{2}}\left[\begin{array}{c}
1+e^{j 2 \pi d_{\lambda} \sin \theta} \\
0 \\
0
\end{array}\right] .
$$

$V_{1}^{-}$is unchanged compared to the previous case, which means, as expected, that the reciprocity theorem is verified for any incidence angle. Now, the difference comes from $V_{2}^{-}$and $V_{3}^{-}$, which are both equal to zero. This means that power is dissipated in the internal loads, but the ratio of the power dissipated to the power captured by the array elements depends on the incidence angle. For instance, no power is 


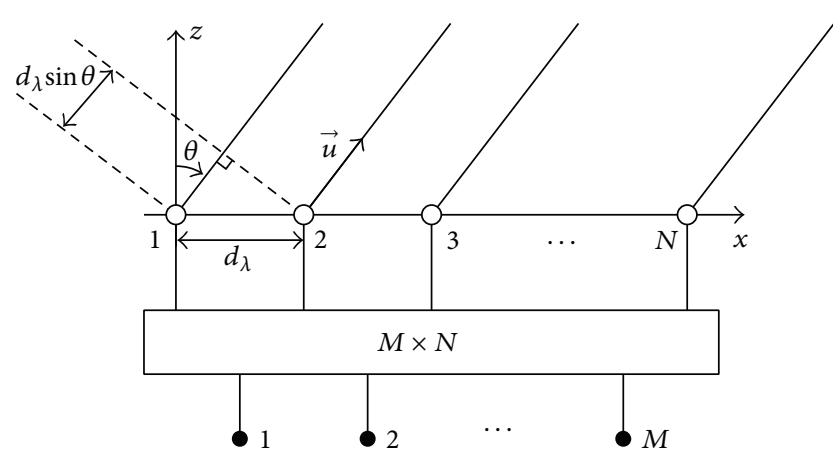

FIGURE 3: Multiple beam $N$-element linear array parameters definition.

dissipated for a normal incidence, while half of the power received is dissipated for the incidence angle $\theta=30^{\circ}$.

If we consider the transfer function only, the two beam forming networks produce exactly the same result in the transmitting and in the receiving mode. Interestingly, the gain variation with the considered angular direction in the transmitting mode is compensated in the receiving mode by either reradiated power (case of a "lossless" beam forming network) or dissipated power (case of a "lossy" beam forming network). In the next section, this property is generalized to any multiple beam forming network.

\section{Generalization to Multiple Beam Forming Networks}

3.1. Multiple Beam Forming Networks Description. Multiple beam forming networks are circuits that generate several simultaneous beams from a same array antenna. Figure 3 illustrates the case considered here of such a network feeding a linear array antenna, although the description could be generalized to any type of array antennas. These networks are $P$-port passive devices having $M$ beams ports (referred to as input ports in the transmitting mode) and $N$ ports connected to the array elements (referred to as output ports in the transmitting mode) such that $M+N=P$ [15]. They are often characterized by their $N \times M$ transfer matrix $T$. The column $m$ of this matrix, with $m$ varying from 1 to $\bar{M}$, corresponds to the excitation coefficient vector $C_{m}$ of the array elements produced by feeding the beam port $m$ in the transmitting mode. The scattering matrix of a multiple beam forming network can then be written as follows:

$$
[S]=\left[\begin{array}{ll}
\underline{I}_{M \times M} & \underline{T}_{M \times N}^{t} \\
\underline{T}_{N \times M} & \underline{O}_{N \times N}
\end{array}\right]_{P \times P},
$$

where the matrices $\underline{I}$ and $\underline{O}$ contain the matching and coupling coefficients of, respectively, the beam ports and the array element ports.

Reciprocity at circuit level imposes the matrix $[S]$ to be symmetric [10], which imposes $\underline{I}$ and $\underline{O}$ to be symmetric but not necessarily $\underline{T}$. Also, $\underline{T}$ is not necessarily a square matrix as the number of input and output ports may differ $(M \neq N)$.
Interestingly, $\underline{T}$ is associated to the transmitting mode while its transpose matrix, $T^{t}$, is associated to the receiving mode.

A typical requirement for antenna design is that all beam ports are matched and decoupled; that is, $I$ is a zero matrix. Theoretically lossless beam forming networks produce orthogonal excitations, resulting in beam patterns orthogonal in space (zero inner product) which impose constraints on beam crossover and side lobe levels [16, 17]. One can easily demonstrate that a lossless beam forming network with matched input and output ports as well as decoupled input ports and decoupled output ports $(\underline{I}$ and $\underline{O}$ are zero matrices) requires $\underline{T}$ to be unitary. In that case, the number of input and output ports must be identical. It was even further demonstrated that imposing the same amplitude distribution to all the beams as well as an arithmetic phase progression (to produce optimal field combination in a given angular direction) leads to a unique matrix solution with necessarily uniform amplitude distribution [18]. Let us now investigate the difference in behavior between the transmitting and receiving modes of such networks and extend the property observed comparing the tee junction and the Wilkinson power divider.

3.2. The Particular Case of a Unitary Transfer Matrix. This particular case is of high interest because it corresponds to a well-known practical implementation: the Butler matrix $[19,20]$. This matrix has been extensively investigated as it is a canonical lossless beam forming network. General design procedures are available in the literature [21, 22]. Interestingly, this specific matrix has similitudes with the Fourier Transform [23, 24]. The Nolen matrix [25], in its square form, can be seen as a generalized form of lossless (unitary) network, which can be reduced to a Butler matrix in the particular case of a matrix size equal to an integral power of two [26]. But the Nolen matrix actually has a topology comparable to the Blass matrix [27], which is based on serial feeding and provides full flexibility on the number of input and output ports. This link between Blass and Nolen matrices was exploited to extend to Nolen matrices a specific Blass matrix design procedure and thus to facilitate Nolen matrix design [28].

In the transmitting mode, a beam forming network characterized by a unitary transfer matrix is lossless because the excitations produced by feeding any beam port are unit vectors (the power supplied at the input port is fully transferred to the output ports). From an antenna point of view, the transfer function in the transmitting mode is defined by the radiation pattern produced by feeding a given beam port, which naturally varies with the observation angle (location of antenna 2). In the receiving mode, as already discussed, the power captured by the array elements does not depend on the plane wave incidence angle. How does reciprocity hold? This can be understood having a look at all the patterns produced by a unitary transfer matrix. As an illustration, we consider a $4 \times 4$ Butler matrix designed with $90^{\circ}$ hybrids, also called a symmetric Butler matrix (due to the symmetrical arrangement of the beams with respect to the normal angular direction), schematically represented in Figure 4 . The 4 beams produced by this matrix are given in 


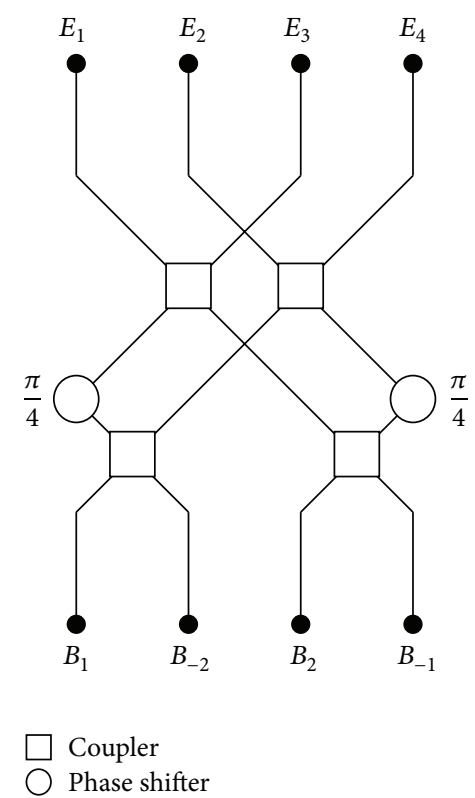

FIGURE 4: $4 \times 4$ symmetrical Butler matrix.

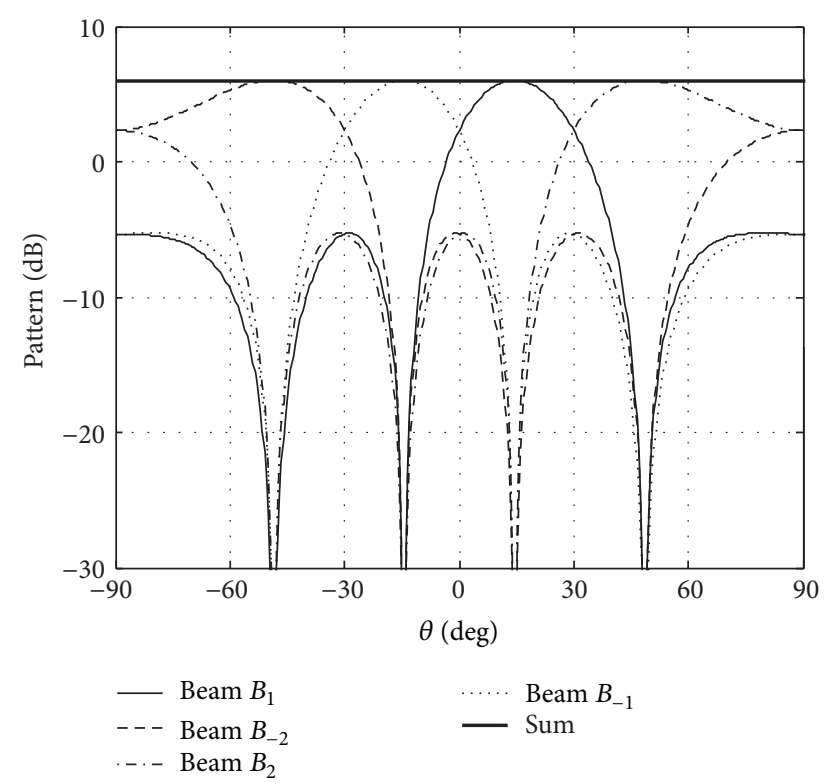

FIGURE 5: Radiation patterns of a $4 \times 4$ symmetrical Butler matrix.

Figure 5 for $d_{\lambda}=0.5$. The peak of the transfer function in the transmitting mode is $6 \mathrm{~dB}$. As highlighted in Figure 5, the sum of the 4 patterns in any angular direction is constant and equal to the maximum of each beam pattern $(6 \mathrm{~dB})$ with contributions from the 4 beam ports varying depending on the observation angle. In the receiving mode, a similar phenomenon appears in the beam forming network. As the plane wave incidence angle varies, the power captured by the array elements is distributed among the beam ports. The power transmitted to a given beam port in the receiving mode as a function of the incidence angle varies as the corresponding beam pattern produced by this same beam port in the transmitting mode. Some particular cases can be highlighted. For instance, when the plane wave incidence angle corresponds to the pointing direction of one of the 4 beams produced, all the power captured by the array elements is directed toward the corresponding beam port. Beam ports are thus decoupled in these particular angular directions [29]. At the crossover between two adjacent beams, the power is distributed equally between the two corresponding beam ports, with a smaller contribution to the two remaining beam ports. This effect has to be taken into consideration if the beams are to be used over a certain beam-width and not only at their peak. Depending on the behavior of the subsystem connected to the beam ports, this undesired cross-coupling may be either reflected by or dissipated in the subsystem connected to the nonrelevant beam ports.

3.3. General Case of a Nonunitary Transfer Matrix. In the general case, the number of beam ports and array element ports are not necessarily equal, resulting in a transfer matrix that is not a square matrix. Consequently, the transfer matrix is no longer unitary and (3) is not verified. The Hermitian inner product of the transfer matrix $\underline{T}$ is as follows:

$$
\begin{aligned}
& \underline{T}^{t} \underline{T}^{*} \\
& =\left[\begin{array}{cccccc}
C_{1} \cdot C_{1}^{*} & C_{1} \cdot C_{2}^{*} & \cdots & C_{1} \cdot C_{m}^{*} & \cdots & C_{1} \cdot C_{M}^{*} \\
C_{2} \cdot C_{1}^{*} & C_{2} \cdot C_{2}^{*} & \cdots & C_{2} \cdot C_{m}^{*} & \cdots & C_{2} \cdot C_{M}^{*} \\
\vdots & \vdots & \ddots & \vdots & & \vdots \\
C_{m} \cdot C_{1}^{*} & C_{m} \cdot C_{2}^{*} & \cdots & C_{m} \cdot C_{m}^{*} & \cdots & C_{m} \cdot C_{M}^{*} \\
\vdots & \vdots & & \vdots & \ddots & \vdots \\
C_{M} \cdot C_{1}^{*} & C_{M} \cdot C_{2}^{*} & \cdots & C_{M} \cdot C_{m}^{*} & \cdots & C_{M} \cdot C_{M}^{*}
\end{array}\right]_{M \times M}
\end{aligned}
$$

The right side of (11) contains the Hermitian inner products of the family of vectors composed by the columns of $\underline{T}$. If this family of vectors is linearly independent, the product reduces to a unit matrix, meaning that a relation equivalent to the "lossless" condition can be found for a transfer matrix that is not necessarily square. Consequently, the transmitting mode associated to $T$ can be lossless. This condition is satisfied if $M \leq N$. From a practical point of view, this means that the number of beam ports is less than the number of array elements. This is often the case, as a large number of elements is usually needed to achieve the directivity required by a given application while a small number of beams enable us to maintain a reasonably low beam forming network complexity.

Let us assume that $T$ is such that its column vectors form a family of linearly independent vectors $(M<N)$. This means that the columns of $T^{t}$ cannot have the same property. In particular, the norm of the column vectors in $\underline{T}^{t}$ is not unity. Based on (10), two solutions are possible for $[S]$. If $\underline{O}$ is a zero matrix, $[S]$ cannot be a unitary matrix and must have internal losses varying with the incidence angle in the receiving mode. The Wilkinson power divider discussed previously is a particular case of this solution. If we 


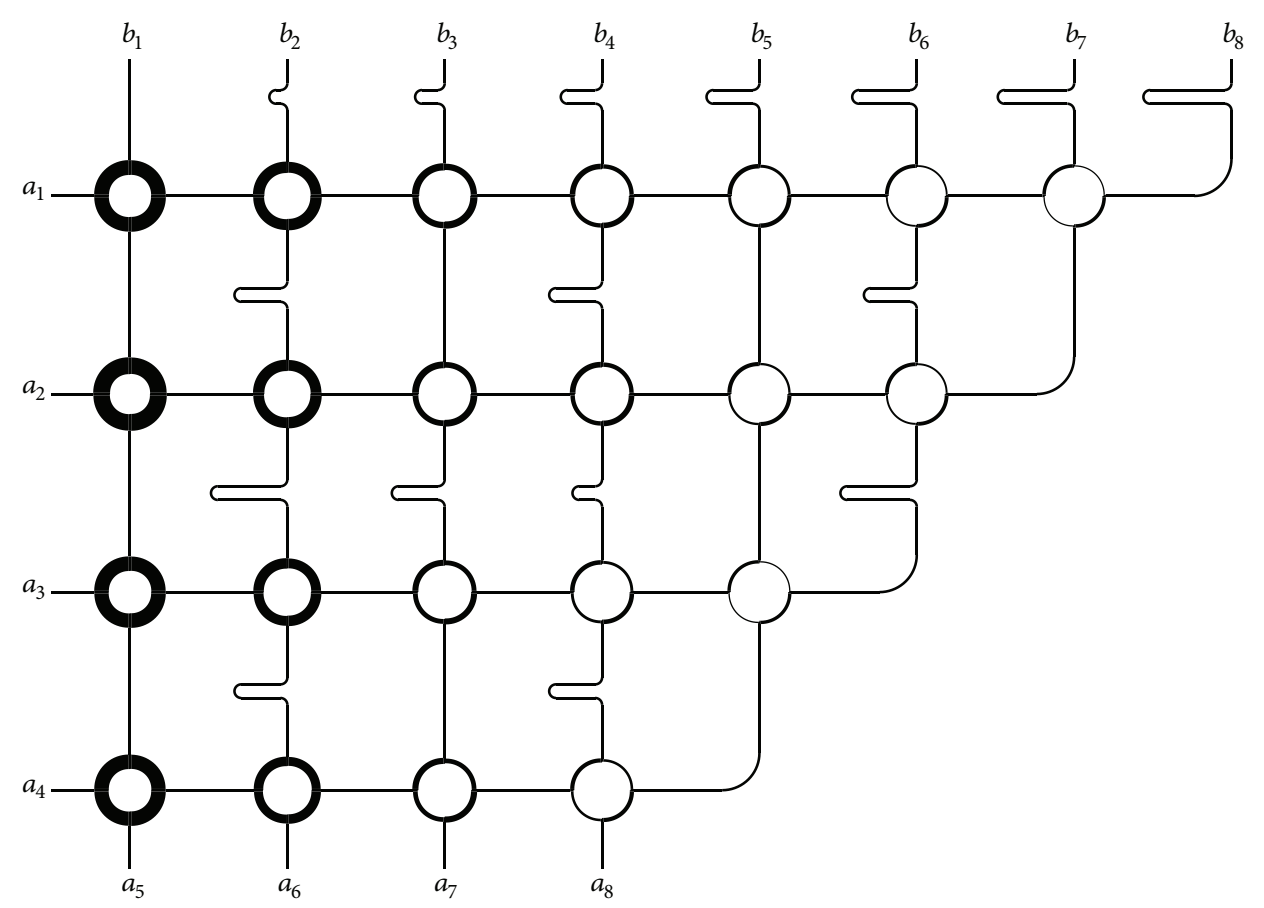

FIgURE 6: Layout of a $4 \times 8$ Nolen matrix with tapered amplitude distribution.

want $[S]$ to be a unitary matrix, $\underline{O}$ cannot be a zero matrix. The coefficients of $\underline{O}$ need to be defined in such a way that the column vectors of the matrix $\left[\frac{T^{t}}{\underline{Q}}\right]_{P \times N}$ form a family of vectors linearly independent. This results in degraded matching and decoupling of the array element ports. The tee junction discussed previously is a particular case of this solution. Obviously, both solutions operate similarly at the overall two-antenna system level, satisfying the reciprocity theorem. The extra power captured by the array elements for certain incidence angles in the receiving mode is in part directed to other beam ports as cross-coupling (same phenomenon as in the case of unitary transfer matrices) and in part internally dissipated in the case of the first solution or reradiated in the case of the second solution. These two solutions can easily be distinguished as one requires internal loads to produce matching and decoupling of the array element ports while the other is only made of reactive components.

Let us illustrate this with a practical example. We consider the $4 \times 8$ Nolen matrix described in $[6,7]$. This design is a specific implementation of the solution originally described for Butler matrices in [30] and taking advantage of the specific topology of Nolen matrices. The original idea was to divide the outputs of a square unitary matrix with out-of-phase power dividers, introducing a $180^{\circ}$ phase shift between signals coming out of a same unitary matrix output port. This enables us to maintain the beam pointing (arithmetic phase progression) while introducing amplitude distribution control (enabling, for example, a Gaussian amplitude distribution to reduce the side lobes). The corresponding Nolen matrix layout is reported in Figure 6. The matrix was designed at $4 \mathrm{GHz}$, using microstrip transmission line technology printed on a substrate by Neltec [31] with reference NY9217, characterized by a dielectric constant of 2.17 and a thickness of $0.254 \mathrm{~mm}$. A copper thickness of $18 \mu \mathrm{m}$ was considered in the simulation as well as tangential losses of 0.0008 as given by the manufacturer. Circular branch line couplers were implemented as their topology is well-suited for Nolen matrices and simple transmission line lengths were used as phase shifters. Further technical details on the design of this specific matrix are given in $[6,7]$. The four "useful" beam ports, labeled $a_{1}$ to $a_{4}$, are on the left side of the matrix. The ports on the top, labeled $b_{1}$ to $b_{8}$, are connected to the array elements. The four ports on the bottom, labeled $a_{5}$ to $a_{8}$, are loaded for proper operation of the matrix. The four beams produced by this matrix are illustrated in Figure 7, using an array factor formulation [14]. As expected, the side lobes are significantly reduced, but the crossover between adjacent beams is also much lower. Interestingly, the sum of the 4 useful beams is not constant with the observation angle, which is consistent with the fact that $[S]$ is not unitary. Consequently, variations of the total radiated power with the observation angle are observed in the transmitting mode. The internal losses as a function of the plane wave incidence angle in the receiving mode are given in Figure 8. As expected due to reciprocity, these internal losses are exactly the image of the radiated power variations in the transmitting mode. For comparison, simulated insertion losses of the proposed design are also displayed on Figure 8. They were obtained using the 3D planar EM simulator Momentum by Keysight Technologies [32], which is based on the Method of Moments. The simulation result is quite in good agreement with theoretical values, the difference coming from the line losses resulting from the use of microstrip technology, and small 


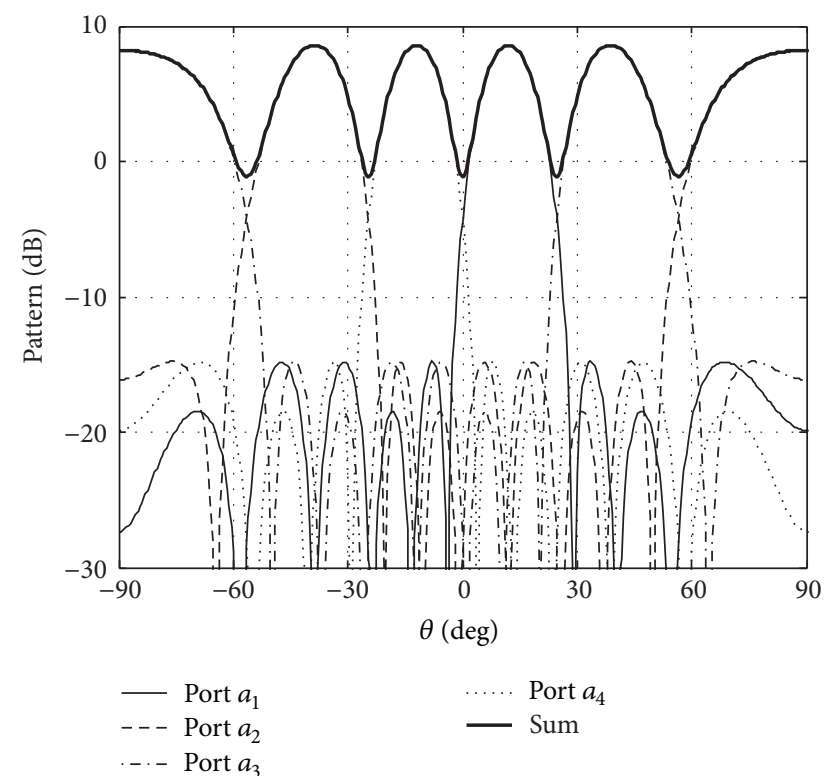

FIgURE 7: Radiation patterns of a $4 \times 8$ Nolen matrix with tapered amplitude distribution.

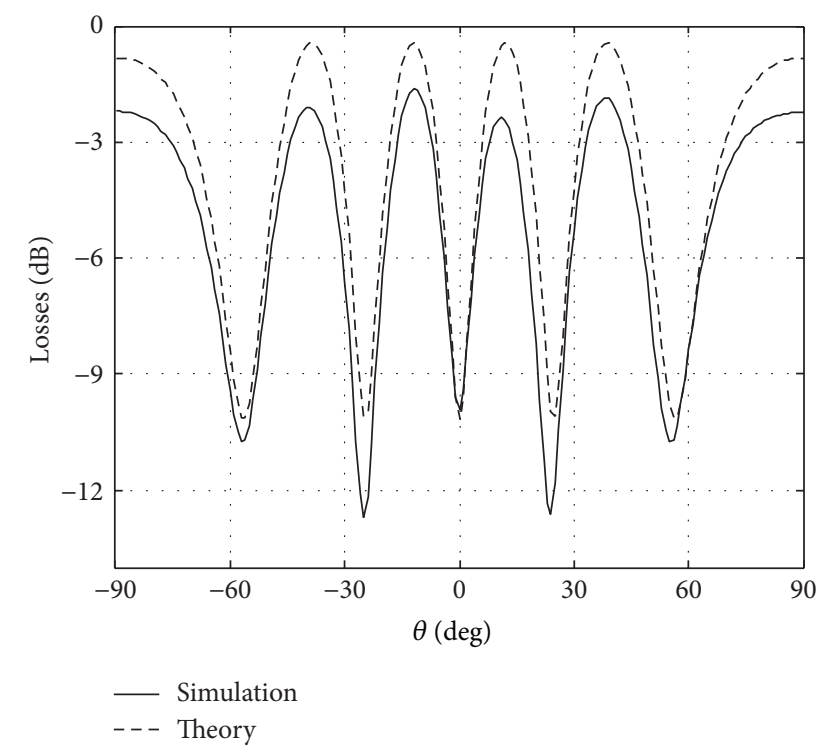

Figure 8: Losses as a function of the plane wave incidence angle in a $4 \times 8$ Nolen matrix operating in the receiving mode.

discrepancies between the theoretical and actual amplitude and phase distributions. As expected, these losses correspond to power dissipated in the loaded ports $a_{5}$ to $a_{8}$. Of course, if the matrix would be considered as an $8 \times 8$ matrix assuming that the loaded ports are also used, then the resulting matrix would be unitary. This is highlighted in Figure 9, displaying the "beams" produced by the loaded ports. Making the summation of all the beams, including those produced by the loaded ports, results in a constant sum power. A similar result could be derived in the case of the Wilkinson power divider if it would be considered as a 5-port device instead of a 3-port device. Obviously, in a practical application case,

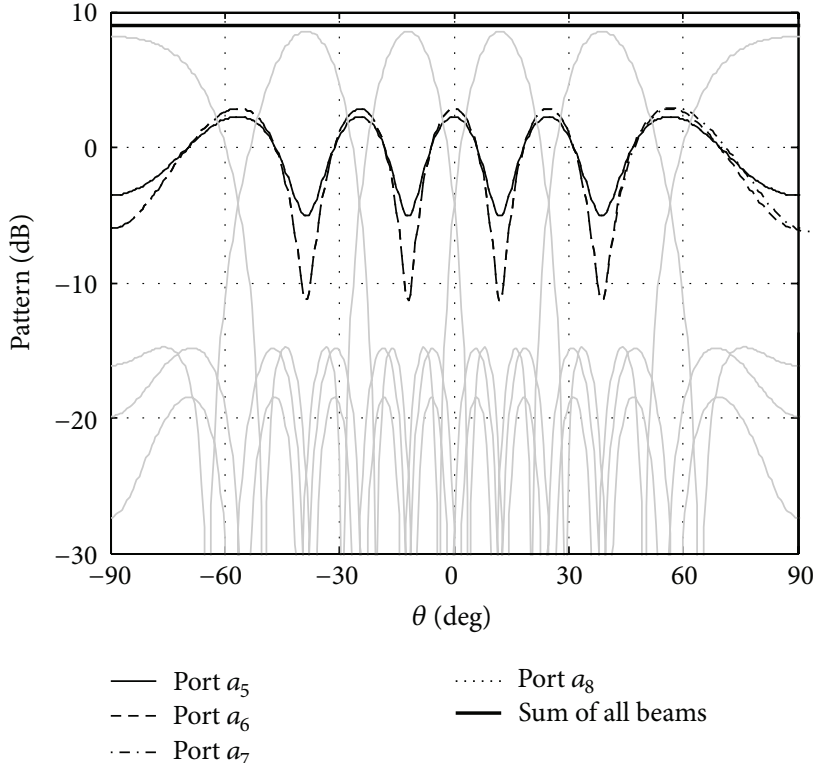

FIGURE 9: Radiation patterns produced by the loaded ports of the considered $4 \times 8$ Nolen matrix with tapered amplitude distribution.

those beams produced by loaded ports are of no use due to their inappropriate pattern shape. An alternative solution to the proposed Nolen matrix design could be to replace by tee junctions the four couplers directly connected to these loaded ports. In that case, the power dissipated in the loads in the receiving mode would instead be reradiated. Another point to highlight about this $4 \times 8$ Nolen matrix design is that the 4 beams produced are not decoupled. Due to a different distribution of the side lobes and corresponding nulls induced by the Gaussian amplitude distribution, the peak gain of one beam is no longer corresponding to nulls of all the other beams. This translates into small losses (about $0.4 \mathrm{~dB}$ ) in the receiving mode even when the plane wave incidence angle coincides with one of the four beam pointing directions. This is easily understood as the beam forming network is designed to produce a Gaussian amplitude distribution, while in the receiving mode, a uniform amplitude distribution is "captured" by the array elements.

This discussion could be extended to another case of multiple beam forming networks: those that are lossy in the transmitting and the receiving modes. A typical example is the Blass matrix [27]. Following the same reasoning, one can easily see that internal losses do not need to be the same in the transmitting and the receiving modes for reciprocity to hold, even in such lossy networks.

\section{Conclusions}

This paper discussed reciprocity and multiple beam forming network properties from an antenna point of view. Differences in behavior were highlighted comparing the transmitting and receiving modes of linear array antennas fed by such networks. Reciprocity is obviously confirmed at antenna level, but this does not imply that the antenna operation 
is the same in the transmitting and receiving modes. As demonstrated, the beam forming network can be lossless in the transmitting mode and lossy in the receiving mode without infringing the reciprocity theorem. Mathematical condition to observe such a phenomenon was discussed in the general case of a $M \times N$ multiple beam forming network. Depending on the selected technical solution, the multiple beam forming network may dissipate power or reradiate it. This difference in behavior has to be well understood and taken into account at an early stage in the antenna design to insure that its behavior is compatible with the targeted application. Typical issues to be considered include mutual coupling or interference with other antenna systems, beam ports cross-coupling, reflections on adjacent structures, power handling and dissipation, and so forth. Understanding the difference in behavior between the transmitting and the receiving modes at antenna level can also help us to define adequate testing set-up and to investigate possible failure effects.

\section{Conflict of Interests}

The author declares that there is no conflict of interests regarding the publication of this paper.

\section{Acknowledgment}

The author would like to thank Dr. Piero Angeletti, from the European Space Agency, for interesting discussions on this topic.

\section{References}

[1] J. van Bladel, "On the equivalent circuit of a receiving antenna," IEEE Antennas and Propagation Magazine, vol. 44, no. 1, pp. 164-165, 2002.

[2] D. Pozar, "Scattered and absorbed powers in receiving antennas," IEEE Antennas and Propagation Magazine, vol. 46, no. 1, pp. 144-145, 2004.

[3] A. W. Love, "Scattering by a paraboloidal reflector antenna: a retraction and an explanation," IEEE Antennas and Propagation Magazine, vol. 46, no. 5, pp. 129-130, 2004.

[4] S. R. Best and B. C. Kaanta, "A tutorial on the receiving and scattering properties of antennas," IEEE Antennas and Propagation Magazine, vol. 51, no. 5, pp. 26-37, 2009.

[5] A. Alù and S. Maslovski, "Power relations and a consistent analytical model for receiving wire antennas," IEEE Transactions on Antennas and Propagation, vol. 58, no. 5, pp. 1436-1448, 2010.

[6] N. J. G. Fonseca and N. Ferrando, "Nolen matrix with tapered amplitude law for linear arrays with reduced side lobe level," in Proceedings of the 4th European Conference on Antennas and Propagation (EuCAP '10), pp. 1-5, Barcelona, Spain, April 2010.

[7] N. J. G. Fonseca, Étude de systèmes micro-ondes d'alimentation d'antennes réseaux pour applications multifaisceaux [Ph.D. thesis], Université de Toulouse-Institut National Polytechnique de Toulouse, Toulouse, France, 2010.

[8] L. Sevgi, "Reciprocity: some remarks from a field point of view," IEEE Antennas and Propagation Magazine, vol. 52, no. 2, pp. 205-210, 2010.
[9] C. A. Balanis, "Antenna theory: a review," Proceedings of the IEEE, vol. 80, no. 1, pp. 7-23, 1992.

[10] D. M. Pozar, "Chapter 4: microwave network analysis," in Microwave Engineering, John Wiley \& Sons, New York, NY, USA, 2nd edition, 1998.

[11] A. T. De Hoop, "A reciprocity relation between the transmitting and the receiving properties of an antenna," Applied Scientific Research, vol. 19, no. 1, pp. 90-96, 1968.

[12] A. T. de Hoop and G. de Jong, "Power reciprocity in antenna theory," Proceedings of the IEE, vol. 121, no. 10, pp. 1051-1056, 1974.

[13] D. M. Pozar, "Chapter 7: power dividers and directional couplers," in Microwave Engineering, John Wiley \& Sons, New York, NY, USA, 2nd edition, 1998.

[14] R. C. Hansen, "Chapter 2: basic array characteristics," in Phased Array Antennas, John Wiley \& Sons, Hoboken, NJ, USA, 2nd edition, 2009.

[15] R. C. Hansen, "Chapter 10: multiple-beam antennas," in Phased Array Antennas, John Wiley \& Sons, Hoboken, NJ, USA, 2nd edition, 2009.

[16] J. Allen, "A theoretical limitation on the formation of lossless multiple beams in linear arrays," IRE Transactions on Antennas and Propagation, vol. 9, no. 4, pp. 350-352, 1961.

[17] W. D. White, "Pattern limitations in multiple-beam antennas," IRE Transactions on Antennas and Propagation, vol. 10, no. 4, pp. 430-436, 1962.

[18] W. Kahn and H. Kurss, "The uniqueness of the lossless feed network for a multibeam array," IRE Transactions on Antennas and Propagation, vol. 10, no. 1, pp. 100-101, 1962.

[19] J. Butler and R. Lowe, "Beam-forming matrix simplifies design of electronically scanned antennas," Electronic Design, vol. 9, pp. 170-173, 1961.

[20] J. Shelton and K. Kelleher, "Multiple beams from linear arrays," IRE Transactions on Antennas and Propagation, vol. 9, no. 2, pp. 154-161, 1961.

[21] H. Moody, "The systematic design of the Butler matrix," IEEE Transactions on Antennas and Propagation, vol. 12, no. 6, pp. 786-788, 1964.

[22] T. Macnamara, "Simplified design procedures for butler matrices incorporating $90^{\circ}$ hybrids and $180^{\circ}$ hybrids," IEE Proceedings H: Microwaves, Antennas and Propagation, vol. 134, no. 1, pp. 50-54, 1987.

[23] J. P. Shelton, "Fast Fourier transforms and Butler matrices," Proceedings of the IEEE, vol. 56, no. 3, p. 350, 1968.

[24] W. H. Nester, "The fast Fourier transform and the Butler matrix," IEEE Transactions on Antennas and Propagation, vol. 16, no. 3, p. 360, 1968.

[25] J. C. Nolen, Synthesis of multiple beam networks for arbitrary illuminations [Ph.D. thesis], Bendix Corporation, Radio Division, Baltimore, Md, USA, 1965.

[26] W. C. Cummings, "Multiple beam forming networks," Technical Note, Lincoln Laboratory, 1978.

[27] J. Blass, "Multidirectional antenna-a new approach to stacked beams," in Proceedings of the IRE International Convention Record, vol. 8, part 1, pp. 48-50, 1966.

[28] N. J. G. Fonseca, "Printed S-band $4 \times 4$ nolen matrix for multiple beam antenna applications," IEEE Transactions on Antennas and Propagation, vol. 57, no. 6, pp. 1673-1678, 2009.

[29] S. Stein, "On cross coupling in multiple-beam antennas," IRE Transactions on Antennas and Propagation, vol. 10, no. 5, pp. 548-557, 1962. 
[30] J. P. Shelton, "Reduced sidelobes for Butler-matrix-fed linear arrays," IEEE Transactions on Antennas and Propagation, vol. 17, no. 5, pp. 645-647, 1969.

[31] http://www.parkelectro.com/.

[32] http://www.keysight.com/. 

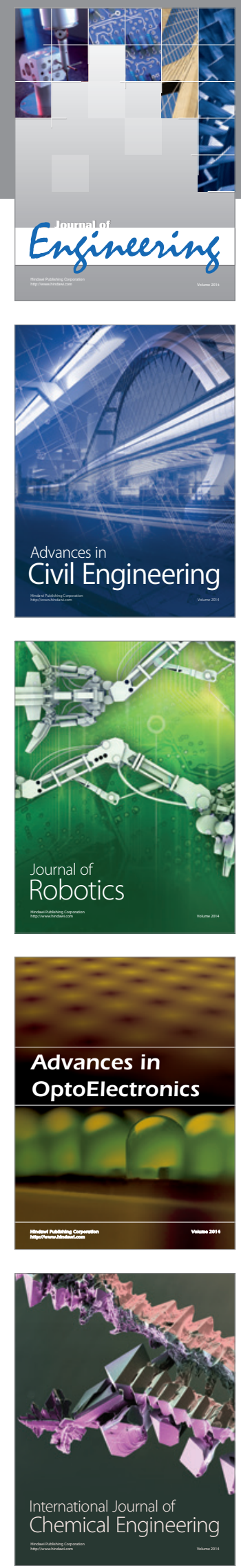

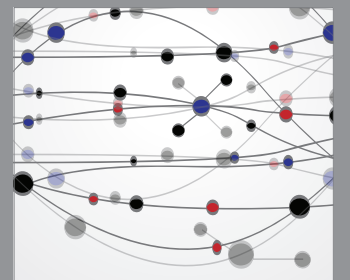

The Scientific World Journal
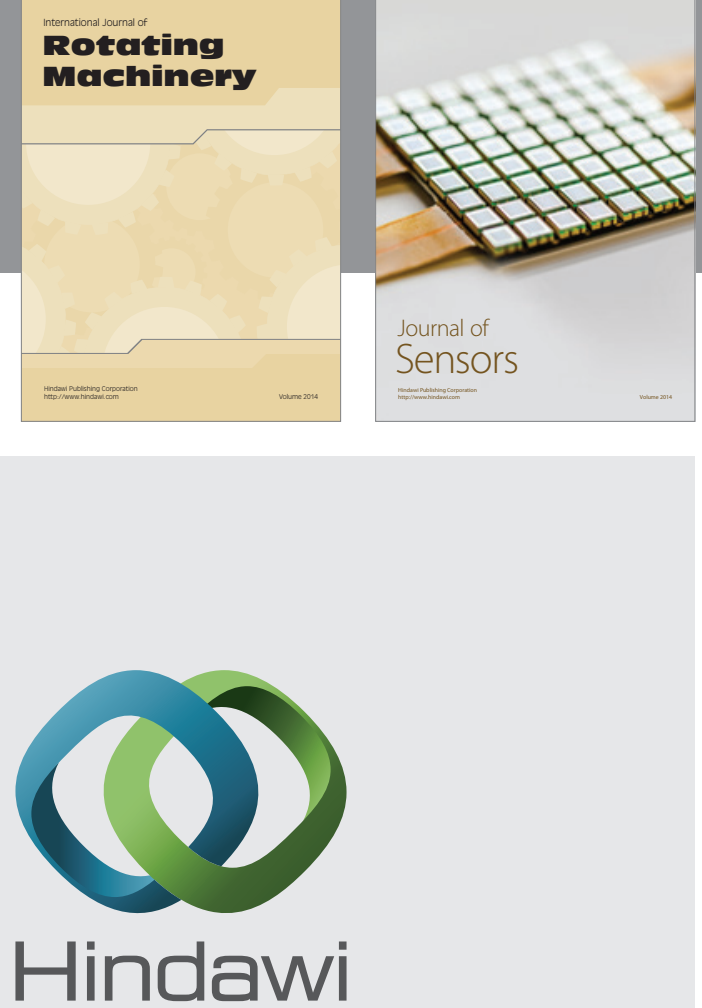

Submit your manuscripts at http://www.hindawi.com
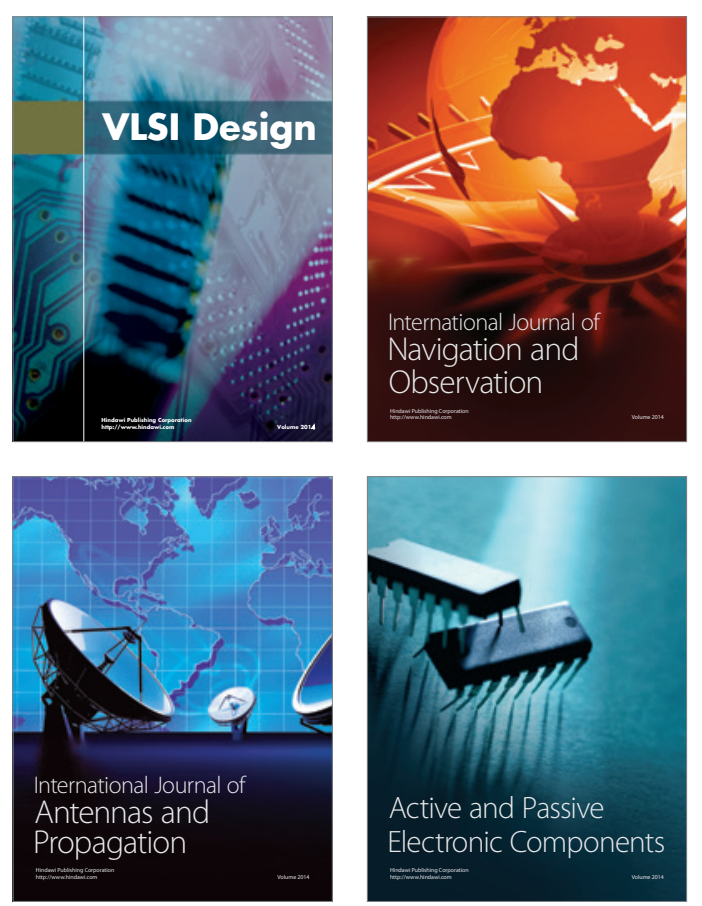
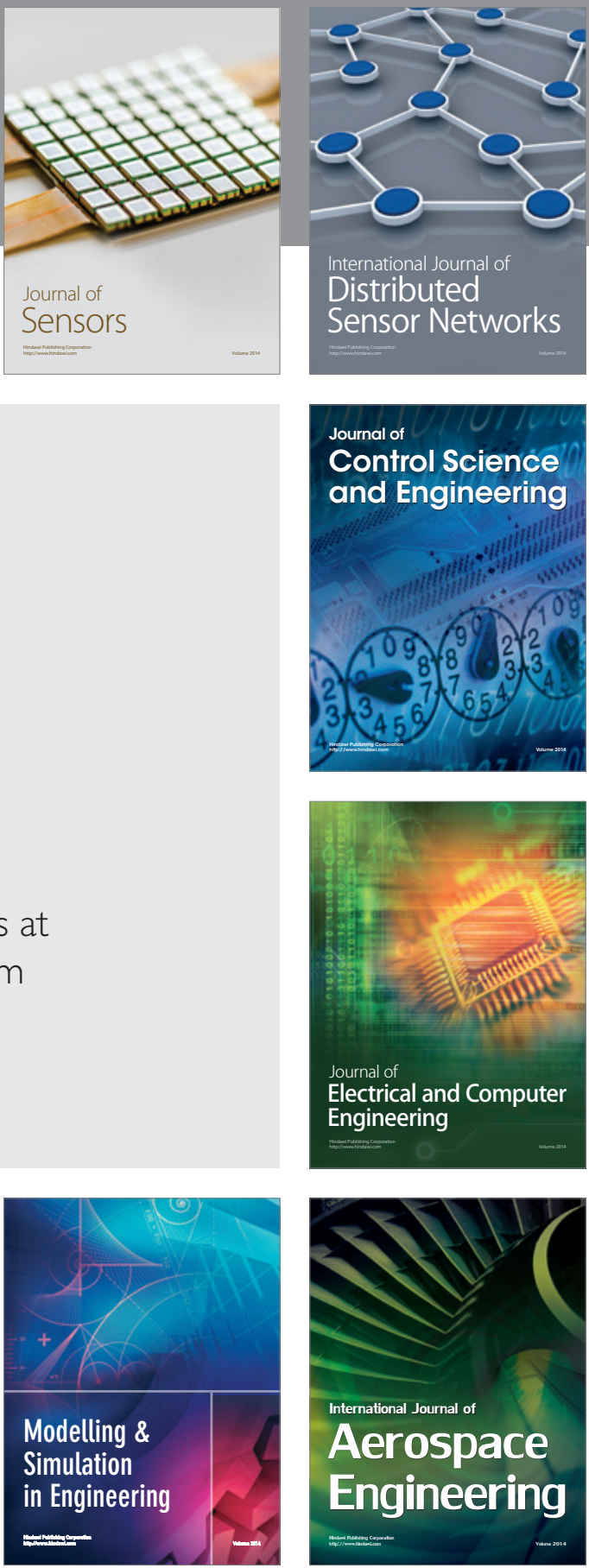

Journal of

Control Science

and Engineering
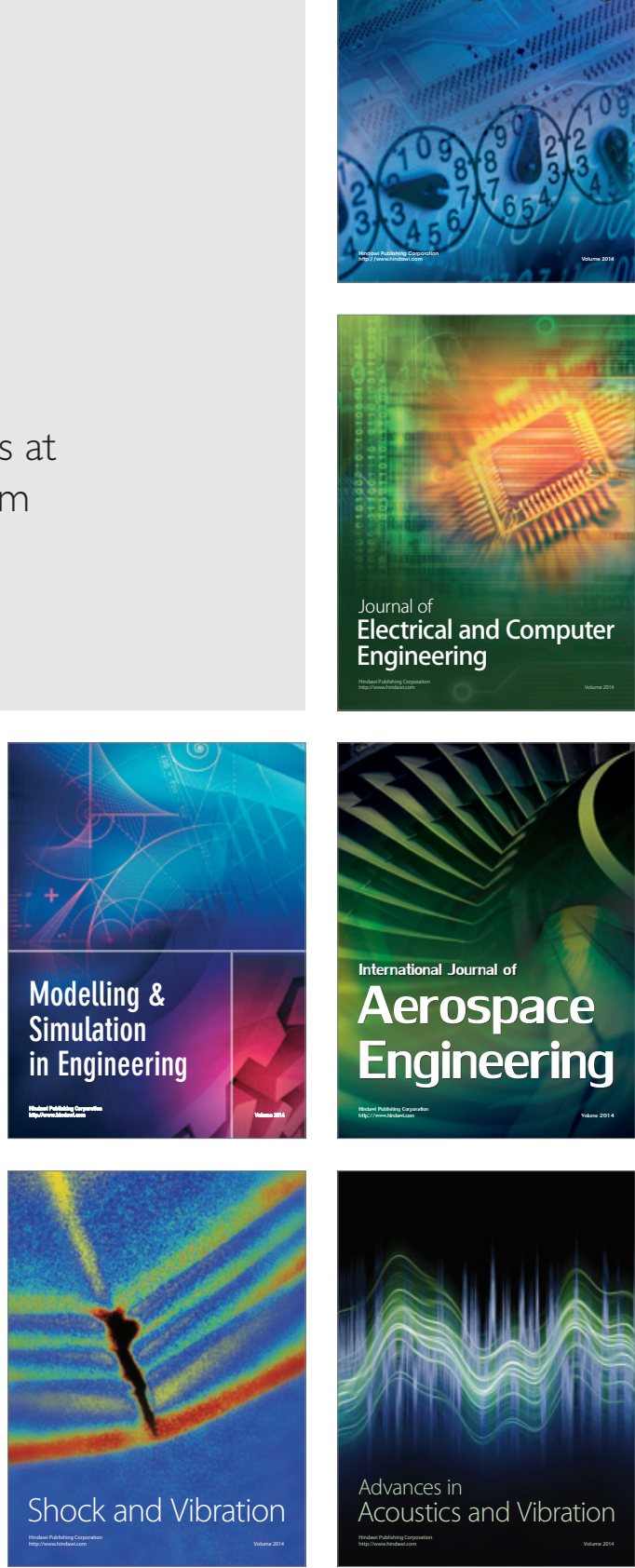